\title{
Short-time dynamics of the helix-coil transition in polypeptides
}

\author{
Everaldo Arashiro* and J. R. Drugowich de Felício ${ }^{\dagger}$ \\ Departamento de Física e Matemática, FFCLRP Universidade de São Paulo, Avenida Bandeirantes 3900, CEP 14040-901, \\ Ribeirão Preto, São Paulo, Brazil \\ Ulrich H. E. Hansmann \\ Department of Physics, Michigan Technological University, Houghton, Michigan 49931-1291, USA
}

(Received 22 June 2005; published 21 April 2006)

\begin{abstract}
We study the critical relaxation of the helix-coil transition in all-atom models of polyalanine chains. We show that at the critical temperature the decay of a completely helical conformation can be described by scaling relations that allow us estimating the pertinent critical exponents. The present approach opens a new way for characterizing transitions in proteins and may lead to a better understanding of their folding mechanism. An application of the technique to the 34-residue human parathyroid fragment $\mathrm{PTH}(1-34)$ supports universality of the helix-coil transition in homopolymers and (helical) proteins.
\end{abstract}

DOI: 10.1103/PhysRevE.73.040902

PACS number(s): 87.15.-v, 64.60.Fr, 64.60.Ht, 87.14.Ee

The process by that a protein folds into its biologically active state is still only partially understood. For instance, folding of proteins involves transitions between different thermodynamic states and the nature of these transitions is an active area of research. Computer experiments provide an important tool but are extremely difficult for detailed protein models [1] that are characterized by a rough energy landscape with their huge number of local minima.

Considerable effort has been put into the development of novel sampling techniques that promise to overcome this socalled multiple minima problem. Successful examples are generalized-ensemble techniques such as parallel tempering [2], a broad-histogram method [3], or multicanonical sampling [4]. However, these methods rely on an artificial dynamics. Here we explore a complementary approach that leaves the "physical" dynamics unchanged and extracts crucial information from the critical relaxation of regular canonical simulations. In that sense it may open a new window on the folding mechanism, a longstanding problem in protein science. The analysis of such short-time dynamics is known as a powerful tool in statistical physics [5-8] but applied here for the first time to proteins.

An important example of folding transitions is the formation of secondary structure elements such as $\alpha$ helices or $\beta$ sheets. In the case of $\alpha$ helices, this process resembles crystallization or melting and has been extensively studied [9]. Evidence was presented in Ref. [10] that the helix-coil transition is for polyalanine a true thermodynamic phase transition. We study in the present paper the nonequilibrium evolution process of $(A l a)_{N}$ chains $(N=10,20$, and 40) in shorttime Monte Carlo (MC) simulations. Our investigation is later extended toward the 34-residue human parathyroid fragment PTH(1-34).

\footnotetext{
*Electronic address: everaldo@pg.ffclrp.usp.br

†Electronic address: drugo@usp.br. Author to whom all correspondence should be addressed.

‡Electronic address: hansmann@mtu.edu
}

During the last years, a better understanding of the critical relaxation process and universality has been achieved. As shown by Janssen et al. [11] universality and scaling behavior are already present in the early times of their evolution. When a system, characterized by an order parameter $m$, is prepared in a macrostate $m_{0}$ at $T>T_{c}$ and quenched to a critical temperature $T=T_{c}$, its time evolution is (after a microscopic time $t_{\text {mic }}$ ) described by a power-law $m(t) \sim t^{\theta}$. This initial small magnetization $m_{0}$ increases during a small macroscopic time $t_{\text {mic }}<t<t_{i}$ before its expected decay toward the equilibrium [7]. The new exponent $\theta$ is independent of the known set of static exponents and of the dynamical critical exponent $z$. Another universal behavior of the dynamic relaxation process can be obtained with an ordered initial state $m_{0}=1$ [12], which decays at the critical temperature $T_{c}$ as $t^{-\beta / \nu z}$. This relation is obtained from the more general scaling form:

$$
m(t, \tau, N) b^{-\beta / \nu} m\left(b^{-z} t, b^{1 / \nu} \tau, b^{-1} N\right),
$$

where $b$ is an arbitrary chain size scaling factor and $\tau$ is the reduced temperature, $\tau=\left(T-T_{c}\right) / T_{c}$. Differentiating $\ln m(t, \tau)$ with respect to the temperature $T$ at $T=T_{c}$,

$$
\left.\frac{\partial \ln m(t, \tau, L)}{\partial \tau}\right|_{\tau=0} \sim t^{1 / \nu z},
$$

leads finally to a scaling relation that allows one to extract the exponent $1 / \nu z$ [13]. Combining the two scaling relations allows one to eliminate the dynamic exponent $z$ and calculate the estimates of the critical exponent $\beta$. Further information on the system can be obtained from the scaling [7] of the second cumulant,

$$
U_{2}(t, L)=\frac{m^{2}(t, L)}{[m(t, L)]^{2}}-1 \sim t^{d / z} .
$$

In the present paper we use Eq. (3) and Eq. (2) to calculate estimates of the exponent $d \nu$. 
The question arises whether the above scaling laws apply also to transitions in proteins. Strictly speaking, phase transitions appear only in infinite systems while proteins are finite systems. However, for a spin system the signal for a phase transition is often found already at a small system size. Similar sharp transitions are observed in proteins. One example is the formation of $\alpha$ helices. In Ref. [14] we have analyzed for the solvated molecule the scaling of the specific heat and found for the helix-coil transition critical exponents compatible with $\alpha=0$ and $\gamma=0$. It is because of these previous results that we decided to choose the helix formation as a test case.

Crucial for our analysis is the definition of an order parameter. Our analog to the magnetization in spin systems is the number of helical residues $q_{H}=2 *\left\langle n_{H}(T)\right\rangle /(N-2)-1$. Here we define a residue as helical if its backbone dihedral angles $(\phi, \psi)$ take values in the range $\left(-70^{\circ} \pm 30^{\circ}\right.$, $-37^{\circ} \pm 30^{\circ}$ ) (their common values in an $\alpha$ helix), and the residue exhibits the hydrogen bonding pattern observed in $\alpha$ helices. The normalization factor $N-2$ ( $N$ the number of residues) is chosen because the flexible terminal residues are usually not part of an $\alpha$ helix. Our definition ensures that $-1 \leqslant q_{H} \leqslant 1$ and $q_{h}\left(T_{c}\right)=0$.

Our short-time MC simulations of the helix-coil transition are based on a detailed, all-atom representation of proteins. The interaction between the atoms is described by a standard force field, ECEPP/2 [15] (Empirical Conformational Energy Program for Peptides, version 2) [15] as implemented in the program package SMMP (Simple Molecular Mechanics for Proteins) [16]. The interactions between our polypeptides and surrounding water are approximated by means of an implicit water model, which assumes that the solvation (free) energy is proportional to the solvent accessible surface area and utilizes the parameter set of Ref. [17] that is often used in conjunction with the ECEPP force field.

We started our investigation by simulating polyalanine chains of length $N=10,20$, and 40 . Our results are averaged over 2000 independent runs for $N=10,20$, and 500 runs for $N=40$. Errors are estimated by dividing these 2000 (500) runs in bins of 100 (50) runs and calculating the fluctuation of the averages obtained for each bin. Figure 1 displays for various temperatures the time series of our order parameter $q_{h}$ as a function of the Monte Carlo time for $A l a_{40}$. We expect to see in such a log-log plot that curves of $q_{H}$ corresponding to low temperatures approach a constant value while hightemperature curves are characterized by an exponential decrease of $q_{H}$. Both temperature regions are separated by the critical temperature $T_{c}$ at which the corresponding curve should be a straight line (in the scaling region). Our plot indicates as critical temperature $T_{c}^{40}=470 \mathrm{~K}$. Similar plots lead to $T_{c}^{10}=315 \mathrm{~K}$ and $T_{C}^{20}=415 \mathrm{~K}$ for $A l a_{10}$ and $A l a_{20}$ (data not shown).

The estimates of the critical temperatures are for $N=10$ and $N=20$ lower than the ones presented in Ref. [14]: $T_{c}^{10}$ $=333(2) \mathrm{K}$ and $T_{c}^{20}=430(2) \mathrm{K}$. Hence, for both chain sizes the estimates obtained from a short-time analysis seems to be lower by $\approx 15 \mathrm{~K}$ than the ones estimated from the peak in specific heat. No value is given in Ref. [14] for $N=40$. Extrapolating the listed values, including $T_{c}^{30}=461(3)$, our value

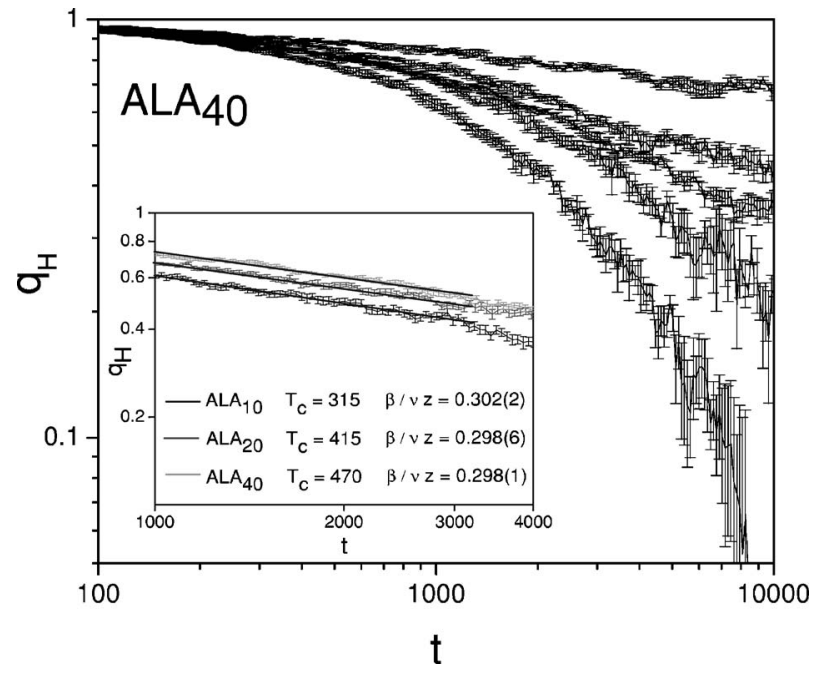

FIG. 1. Log-log plot of the time series of the helical order parameter $q_{H}$ (defined in the text) as a function of Monte Carlo time for polyalanine chains of length $N=40$. The temperatures are (from top to bottom): $T=420,460,470,480 \mathrm{~K}$ and $T=500 \mathrm{~K}$. The inset displays $q_{H}$ for all three polyalanine chains $(N=10,20$, or 30$)$ as measured at their respective critical temperatures. Shown are the data in the scaling region and the best fit through them.

of $T_{c}^{40}=470 \mathrm{~K}$ seems reasonable. Note that for a small system size the the critical temperature as derived from the change in magnetization differs in spin systems also often from the peak in specific heat. Only for large system size is it expected that both values converge. We finally remark that our numbers are calculated from a set of 2000 (500 for $N$ $=40$ ) independent runs while the results of multicanonical runs in Ref. [14] only rely on order $\approx 10$ independent events.

Note that the scaling is similar for all chains. This can be seen from the inset of Fig. 1, which displays for $T=T_{c}$ the time series of the order parameter for all three chain lengths. We focus here on the linear range and show both the measured data points and the straight line that best fits these data. The data for the different chain sizes are almost parallel to each other. From the apparently size-independent slope of these lines, we can obtain an estimate of $\beta / \nu z$. Measuring, in addition, the slope for slightly higher (lower) temperatures and calculating the numerical derivative leads, in addition, to an estimate for $1 / \nu z$. Finally, estimates of the ratio $d / z$ can be extracted from the scaling of $U_{2}(t, L)$, which is shown for the three polyalanine molecules in Fig. 2. Table I lists the values of all three ratios $\beta / \nu z, 1 / \nu z$, and $d / z$, together with the exponents $\beta$ and $d \nu$ extracted from them.

In Ref. [14] it was found that $\alpha=\gamma \approx 0$ and $d \nu \approx 2$. This implies $\beta \approx 1$. Our values for the critical exponent $\beta \approx 0.4$ in Table I are significantly lower than one, but clearly exclude also $\beta=0$, the value expected for a first order transition. A similar statement holds for $d \nu \approx 1.4$ that is much smaller than two, but also excludes a first-order transition $(d \nu=1)$. Note also that the error bars in Ref. [14] are large and do not exclude our estimates of $\beta$ and $d \nu$. Hence, our data support the claim in Ref. [14] that the helix-coil transition in alanine is second-order like. This is different in the gas phase, where the helix-coil transition is of first order [10]. 


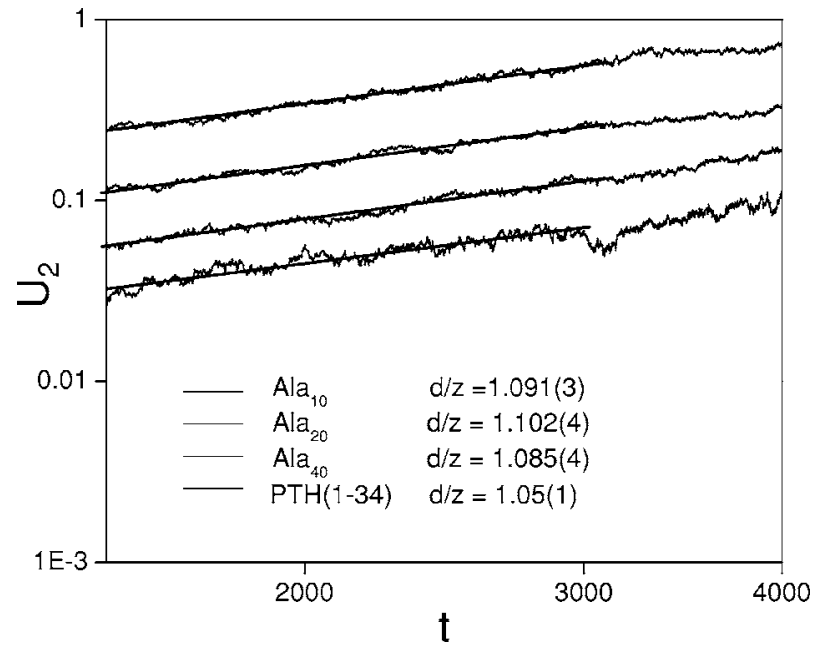

FIG. 2. Log-log plot of the time series of the second moment $F_{2}(t, L)$ for polyalanine chains of length $N=10,20$, or 30 and the polypeptide PTH(1-34) as measured at their respective critical temperatures. Shown are the data in the scaling region and the best fit through them.

Up to this point we have studied the helix-coil transition in a system where critical exponents can be determined by other techniques, as well. This allowed us to compare our results with previous work, and we found qualitative agreement. However, unlike homopolymers, proteins have unique sequences. Hence, an extrapolation to infinite chain length is not a meaningful concept, and a finite size scaling analysis is not possible. In this sense, short-time analysis opens a way to characterize transitions in proteins, too.

We have checked this assumption for the peptide fragment PTH(1-34) corresponding to residues 1-34 of human parathyroid hormone $[18,19]$. The structure of PTH(1-34) has been resolved both crystallized [18] and in solution [19] and is at room temperature almost completely helical. In previous work [20], using the same energy function it was shown that PTH(1-34) exhibits at $T=560(10) \mathrm{K}$ a sharp transition between a high temperature region where disordered coil structures prevail, and a low temperature region that is characterized by mostly helical structures. The nature of this helix-coil transition could not be established in Ref. [20]. Here, we revisit this peptide and study now the short-time dynamics of this helix-coil transition. Our data were obtained for temperatures $T=500,520,530,540,550$, and $560 \mathrm{~K}$ and

TABLE I. Exponents as obtained for polyalanine chains of length $N=10,20$, and 40 from the scaling relations of Eqs. (1)-(3). The last two columns list the critical exponents $\beta$ and $d \nu$, as calculated from these quantities. The last row summarizes the results for the polypeptide PTH(1-34).

\begin{tabular}{cccccc}
\hline \hline$N$ & $\beta / \nu z$ & $1 / \nu z$ & $d / z$ & $\beta$ & $d \nu$ \\
\hline 10 & $0.30(1)$ & $0.767(4)$ & $1.1(1)$ & $0.40(1)$ & $1.42(1)$ \\
20 & $0.30(1)$ & $0.779(9)$ & $1.1(1)$ & $0.38(1)$ & $1.41(1)$ \\
40 & $0.30(2)$ & $0.78(1)$ & $1.1(2)$ & $0.38(1)$ & $1.40(1)$ \\
PTH & $0.22(3)$ & $0.68(1)$ & $1.1(3)$ & $0.33(1)$ & $1.54(1)$ \\
\hline \hline
\end{tabular}

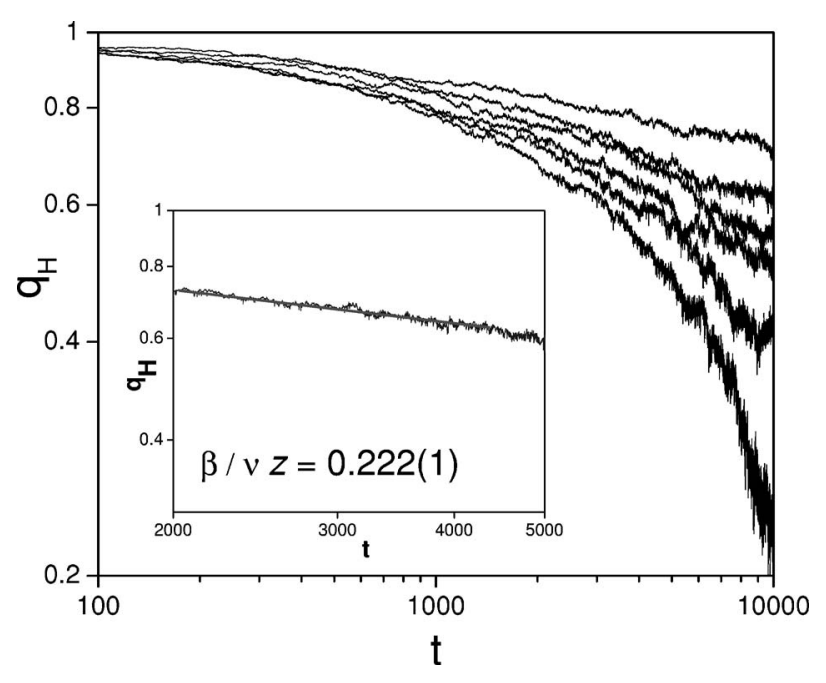

FIG. 3. Log-log plot of the time series of the helical order parameter $q_{H}$ as a function of Monte Carlo time for the polypeptide PTH(1-34) measured at temperatures $T=500,520,530,540,550$, and $560 \mathrm{~K}$. The inset shows the data for the critical temperature $T_{c}=540$ in the scaling region, with the best fit also drawn through them.

rely on 150 runs. Figure 3 displays our order parameter for those temperatures as a function of the Monte Carlo time. From the plot we estimate that the critical temperature is $T$ $=540(10) \mathrm{K}$, a value that is again slightly smaller than the obtained from the peak in specific heat. The scaling region is shown for this temperature in the inset, with both the data point and the best straight-line fit though them. From this fit we obtain exponents $\beta / \nu z$ and $1 / \nu z$ (listed in Table I) that are slightly smaller than the ones obtained for polyalanine, while the ratio $d / z$ agrees for both molecules. Correspondingly, our value for the critical exponent $\beta=0.33(1)$ and $d \nu$ $=1.54(1)$ are comparable with $\beta=0.38(1)$ and $d \nu=1.40(1)$, the values found for polyalanine. These exponents indicate again a second-order transition albeit its folding into its helical structure (where it is biologically active) is quick and appears to have two-state character. The similarity of the exponents suggests "universality" of the helix-coil transition in homopolymers and (helical) proteins.

In summary, we have studied the short-time dynamics of helix-coil transitions in polypeptides. At the critical temperature, the decay of a completely ordered state can be described by scaling relations involving certain (critical) exponents. Comparing for polyalanine, these exponents with the ones obtained by other methods we find qualitative agreement. Similar exponents are also found for PTH(1-34), a polypeptide with a unique sequence for which finite-size scaling is not a meaningful concept. The close values of the critical exponents suggests "universality" of the helix-coil transition in protein-like polymers. These results indicate that the analysis of short-time dynamics is not restricted to spin systems, but of a much larger applicability. Especially, it adds a valuable new tool to the investigation of the folding mechanism in proteins, a longstanding problem in biophysics. 


\section{ACKNOWLEDGMENTS}

E.A. and J.R.D. gratefully acknowledge support by FAPESP (Brazil), and U.H.E.H. by the National Science
Foundation (CHE-0313618). We thank N. A. Alves for stimulating discussions and help in the initial stages of this work.
[1] U. H. E. Hansmann, Comput. Sci. Eng. 5, 64 (2003).

[2] K. Hukushima and K. Nemoto, J. Phys. Soc. Jpn. 65, 1604 (1996); G. J. Geyer, Stat. Sci. 7, 437 (1992).

[3] P. M. C. de Oliveira, T. J. P. Penna, and H. J. Herrmann, Braz. J. Phys. 26, 677 (1996); P. M. C. de Oliveira, Int. J. Mod. Phys. C 9, 497 (1998).

[4] B. A. Berg and T. Neuhaus, Phys. Lett. B 267, 249 (1991).

[5] Z. B. Li, L. Schülke, and B. Zheng, Phys. Rev. Lett. 74, 3396 (1995).

[6] K. Okano, L. Schülke, K. Yamagishi, and B. Zheng, Nucl. Phys. B 485, 727 (1997).

[7] B. Zheng, Int. J. Mod. Phys. B 12, 1419 (1998).

[8] T. Tomé and J. R. Drugowich de Felício, Mod. Phys. Lett. B 12, 873 (1998).

[9] D. Poland and H. A. Scheraga, Theory of Helix-Coil Transitions in Biopolymers (Academic, New York, 1970).

[10] N. A. Alves and U. H. E. Hansmann, Phys. Rev. Lett. 84, 1836 (2000).

[11] H. K. Janssen, B. Schaub, and B. Schmittmann, Z. Phys. B:
Condens. Matter 73, 539 (1989).

[12] D. Stauffer, Physica A 186, 197 (1992).

[13] Z. Li, L. Schülke, and B. Zheng, Phys. Rev. E 53, 2940 (1996).

[14] Y. Peng, U. H. E. Hansmann, and N. A. Alves, J. Chem. Phys. 118, 2374 (2003).

[15] M. J. Sippl, G. Némethy, and H. A. Scheraga, J. Phys. Chem. 88, 6231 (1984), and references therein.

[16] F. Eisenmenger, U. H. E. Hansmann, Sh. Hayryan, and C.-K. Hu, Comput. Phys. Commun. 138, 192 (2001).

[17] T. Ooi, M. Obatake, G. Nemethy, and H. A. Scheraga, Proc. Natl. Acad. Sci. U.S.A. 8, 3086 (1987).

[18] L. Jin, S. L. Briggs, S. Chandrasekhar, N. Y. Chirgadze, D. K. Clawson, R. W. Schevitz, D. L. Smiley, A. H. Tashjian, and F. Zhang, J. Biol. Chem. 275, 27238 (2000).

[19] U. C. Marx, K. Adermann, P. Bayer, W.-G. Forssmann, and P. Rösch, Biochem. Biophys. Res. Commun. 267, 213 (2000).

[20] U. H. E. Hansmann, J. Chem. Phys. 120, 417 (2004). 